\title{
Distortion Analysis and Equivalent Impedance Estimation of a Class-D Full-Wave Rectifier
}

\author{
M. C. Piccirilli, A. Reatti, F. Corti \\ DINFO Via Santa Marta 3 I-50138 Florence, Italy \\ (mariacristina.piccirilli)(alberto.reatti)(fabio.corti)@unifi.it \\ P. De La Pierre, A. Nepote, \\ Magneti Marelli S.p.A., Viale Carlo Emanuele II 150, \\ 10078, Venaria Reale (TO), Italy \\ (piero.delapierre)(andrea.nepote)@magnetimarelli.com
}

\author{
Marian K. Kazimierczuk, A. Ayachit \\ Electrical Engineering - Wright State University \\ 3640 Colonel Glenn Hwy, \\ Dayton, OH 45435, USA \\ (ayachit.2)(marian.kazimierczuk)@wright.edu
}

\begin{abstract}
The substantial increasing of switching devices utilization such as switching power converters, inverters, electronic ballast, UPS, and computer equipment, introduce harmonic distortion in supply systems because of their non-linear characteristics. The waveform distortion produces harmonics that not only increases power losses but also introduces a distortion power, which sums to time-average real and reactive power usually considered in linear circuits. Therefore, effects on harmonics must be considered in the circuit design. In this paper, the current distortion introduced by a Class-D full wave resonant rectifier used in a series-series compensation circuit for wireless power transfer is considered and a general approach to derive a rectifier equivalent impedance that consider the effect of distortion on the output power is presented.
\end{abstract}

Keywords-Harmonic; Dirstortion; Wireless Power Transfer; Resonant Converter

\section{INTRODUCTION}

Nowadays, most of the loads connected to the supply network have a non-linear V-I characteristic [1]. This behavior is usually caused by the switching nature of these devices which introduce harmonics to the electric supply network distorting the current or the voltage waveforms [2]-[4]. Harmonics at high frequencies not only increase the conductor resistance due to skin effect and, more in general, power losses, but also require a approach to the circuit analysis and design. If the voltage and/or current waveforms have a high number of harmonics, the apparent power increases; not only real-time average and reactive power are enough to describe the circuit operation, but also distortion power must be considered [5]. In this paper, the current distortion introduced by a Class-D full wave resonant rectifier used in a series-series compensation circuit for wireless power transfer is studied [6]-[21]. A classical method utilized to model class-D rectifier derives of the rectifier input resistance and this approach is based on two assumptions: $i$ ) the rectifier input current is sinusoidal; $i i)$ the rectifier input voltage is a rectangular waveform and only its fundamental component can be considered [6], [7] and [8]. This method results in a simple and straightforward design procedure because derived equivalent circuit is linear and time-invariant which is reliable until the cited assumptions keep valid. When the DC load resistance increases, however, the real circuit driving the rectifier supplies the rectifier with non-sinusoidal currents [1]. For light loads, the power delivered by the circuit driving the rectifier (e.g. a class-D resonant inverter) must provide a power higher than that derived using the method given in [6], [7] and [8].

The purposes of this paper are as follows: a) analyze the problem of current distortion utilizing power definition in agreement with 1459-2000 IEEE Standard; b) show the load ranges where method given in [6], [7], and [8]; c) introduce a method to derive a rectifier "non-linear" equivalent input resistance, which utilized as inverter load, allows the actual power the inverter must supply, to be calculated, by taking into account the effect of distortions.

\section{MATHEMATICAL FormULATION OF POWER UNDER DISTORTION}

\section{A. Power Components Under Distorted Current and Voltage Operation and IEEE Standard 1459-2000}

In order to measure the distortion of a signal $y(t)$, the total harmonic distortion THD were introduced in the standard IEC 61100. Under the assumption that current and voltage waveforms are periodical with a time-period $T$, and satisfy Dirichlet conditions that is: $i$ ) the waveform has a finite number of discontinuities inside one period; $i i$ ) it has a finite number of relative maximum and minimum points; iii) its integral over one period exists and has a finite value, we have

$$
y(t)=A_{0}+\sum_{k=1}^{\infty} A_{k} \cos \left(k \omega t+\alpha_{k}\right)
$$

where $A_{0}$ is the value of the DC component, $A_{k}$ is the amplitude of the $k$-th harmonic, $\alpha_{k}$ is the initial phase angle of the $k$-th harmonic, $\omega=2 \pi f=2 \pi / T$ is the angular frequency, $T$ is the period, and $f$ is the frequency of the fundamental waveforms.

IEEE Standard 1459-2000 neglects the current and voltage DC components as, actually, occurs, in any waveform symmetrical with respect to the horizontal axis, considers the current and voltage RMS values as follows: 


$$
\begin{aligned}
& I=\sqrt{\frac{1}{T} \int_{0}^{T} i^{2}(t) d t}=\sqrt{I_{1}^{2}+I_{H}^{2}}=\sqrt{I_{1}^{2}+\sum_{k=2}^{\infty} I_{k}^{2}} \\
& =I_{1} \sqrt{1+\frac{\sum_{k=2}^{\infty} I_{k}^{2}}{I_{1}^{2}}}=I_{1} \sqrt{1+T H D_{I}^{2}}
\end{aligned}
$$

and

$$
\begin{aligned}
& V=\sqrt{\frac{1}{T} \int_{0}^{T} v^{2}(t) d t}=\sqrt{V_{1}^{2}+V_{H}^{2}}=\sqrt{V_{1}^{2}+\sum_{k=2}^{\infty} V_{k}^{2}} \\
& =V_{1} \sqrt{1+\frac{\sum_{k=2}^{\infty} V_{k}^{2}}{V_{1}^{2}}}=V_{1} \sqrt{1+T H D_{V}^{2}}
\end{aligned}
$$

In above equations, " 1 " refers to the fundamental components, and " $k$ " to the $k$-th harmonics, and THD I and $\mathrm{THD}_{\mathrm{V}}$ are the current and voltage Total Harmonic Distortions, respectively. IEEE Standard 1459-2000 does not consider the DC component $I_{O} V_{O}$ and defines time-average real power as

$$
P_{1}=V_{1} I_{1} \cos \varphi_{1}
$$

the "harmonic" active power as

$$
P_{H}=\sum_{k=2}^{\infty} P_{k}=\sum_{k=2}^{\infty} V_{k} I_{k} \cos \varphi_{k}
$$

and the total active power as

$$
P=P_{1}+P_{H}
$$

Similarly, the total reactive power $Q_{H}$ is defined as

$$
Q=Q_{1}+Q_{H}=V_{1} I_{1} \sin \varphi_{1}+\sum_{k=2}^{\infty} V_{k} I_{k} \sin \varphi_{k} .
$$

The non-active power $N$ and the distortion power $D$ are

$$
\begin{gathered}
N=\sqrt{S^{2}-P^{2}} \\
D=\sqrt{N^{2}-Q^{2}}=\sqrt{S^{2}-P^{2}-Q^{2}} \\
=\sqrt{\sum_{k=1}^{\infty} \sum_{\substack{h=1 \\
h \neq k}}^{\infty} V_{k}^{2} I_{k}^{2}+V_{h}^{2} I_{h}^{2}-2 V_{k} V_{h} I_{k} I_{h} \cos \left(\varphi_{k}-\varphi_{h}\right)} .
\end{gathered}
$$

\section{B. Power Factor and Power Vectors}

The power factor is defined as

$$
P F=\frac{P}{S}=\frac{P}{V I}
$$

where $I$ and $V$ are the RMS values of current and voltage and $S$ is the apparent power. While in linear circuits operated under no distortion it is simply given as $P F=\cos \varphi_{1}$, in distorted circuit the current and voltage distortions must be considered introducing the $T H D_{I}$ and $T H D_{V}$. Substitution of (2) and (3) into (10) results in

$$
P F=\frac{P}{S}=\frac{P}{V I}=\frac{P}{S_{1}} \times \frac{1}{\sqrt{1+T H D_{I}^{2}} \sqrt{1+T H D_{V}^{2}}}
$$

If the voltage waveform is assumed to be not distorted $V=V_{1}$, $\mathrm{THD}_{\mathrm{V}}=0, P=P_{l}$ and $Q=V I_{1} \sin \varphi_{1}$ and (11) reduces to

$$
\begin{aligned}
& P F=\frac{P}{S}=\frac{P_{1}}{V I}=\frac{P_{1}}{S_{1}} \times \frac{1}{\sqrt{1+T H D_{I}^{2}}} \\
& =\cos \varphi_{1} \times \frac{1}{\sqrt{1+T H D_{I}^{2}}}=D F \times P F_{\text {distorted }}
\end{aligned}
$$

where $D F=\cos \varphi_{1}$ is the displacement factor and $P F_{\text {distorted }}$ is the power factor due to current and voltage distortions. In such a case, as shown in Fig. 1, it is possible to plot an extension of the $2 \mathrm{D}$ power triangle which is given by the "bold" arrows. If current and voltage waveforms are not distorted, the complex power is

$$
\bar{S}_{1}=P+j Q=S_{1} e^{j \varphi_{1}} .
$$

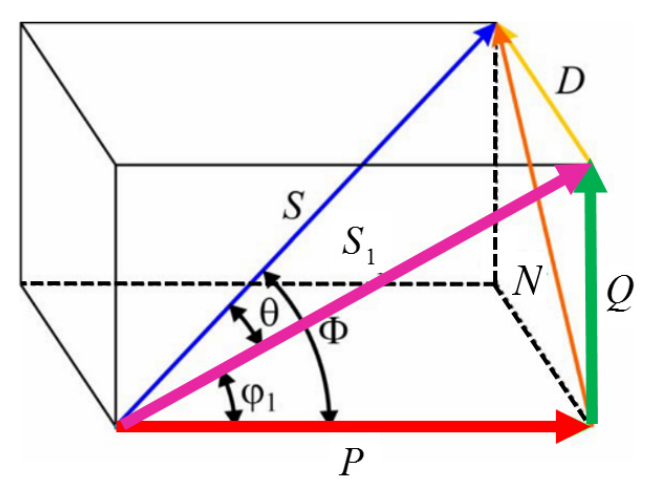

Fig. 1. Vectorial relationship between non Time-Average Real Power (red) associated to the first harmonic, Reactive Power (green) due to the first harmonic, Apparent Power without distortion (magenta), Distortion Power (yellow), Non-Active Power (orange), Apparent Power with distortion (light blue), when $\mathrm{THD}_{\mathrm{V}}=0$.

However, if distortion affects at least current or voltage waveforms, power vector cannot be represented on a $2 \mathrm{D}$ plot only and the actual apparent power is

$$
\bar{S}=\overline{S_{1}}+\bar{D}=\bar{i} P+\bar{j} Q+\bar{k} D
$$

where $\bar{i}, \bar{j}$, and $\bar{k}$ are $P, Q$, and $D$, vectors, respectively.

\section{Class-D RECTIFIER UNDER Distorted OPERATION}

\section{A. Rectifier Analysis with Pure-Sinusoidal Input Current}

To convert AC to DC current, a Class-D full-wave rectifier is used, as shown in Fig. 2(b). Because of diode switching and a large output capacitance, the input voltage is a square wave with a 0.5 duty-cycle with a zero DC component, as shown in Fig. 3. According to the first harmonic assumption, the rectifier is excited by a sinusoidal input current

$$
i_{2}=I_{R m} \sin \omega t
$$

where $I_{R m}$ is the amplitude of $i_{2}$. The current through D1 is 


$$
I_{D 1}=\left\{\begin{array}{cl}
I_{R m} \sin \omega t & 0 \leq \omega t<\pi \\
0 & \pi \leq \omega t<2 \pi
\end{array}\right.
$$

Hence, the DC component of the output current is

$$
\begin{aligned}
& I_{O}=\frac{1}{2 \pi} \int_{0}^{2 \pi}\left(i_{D 1}+i_{D 2}\right) d(\omega t) \\
& =\frac{I_{R m}}{\pi} \int_{0}^{\pi} \sin \omega t d(\omega t)=\frac{2 I_{R m}}{\pi}
\end{aligned}
$$

If the diode is assumed to be ideal, the rectifier input voltage is a square wave expressed by

$$
v_{R}=\left\{\begin{array}{cl}
n V_{O} & 0 \leq \omega t<\pi \\
-n V_{O} & \pi \leq \omega t<2 \pi
\end{array}\right.
$$

where $n$ is the turn-ratio of the transformer shown in Fig. 2(a); in this paper analysis $n=1$ is assumed. The amplitude of the fundamental component of the input voltage is

$$
V_{R m 1}=\frac{1}{\pi} \int_{0}^{2 \pi} v_{R} \sin \omega t d(\omega t)=\frac{2}{\pi} \int_{0}^{\pi} V_{O} \sin \omega t d(\omega t)=\frac{4 V_{O}}{\pi} \text {. }
$$

and the rectifier input resistance is

$$
R_{i}=\frac{V_{R 1 m}}{I_{R m}}=\frac{8}{\pi^{2}} \frac{V_{O}}{I_{O}}=\frac{8}{\pi^{2}} R_{L}
$$

\section{B. Series-Series Compensation Circuit Analysis}

Fig. 2(a) shows a series-series compensation topology for wireless power transfer (WPT), including a Class-D full-wave rectifier. The series-series (SS) compensation topology is widely used because it is not affected by load variations or mutual inductance. Therefore, SS compensation is applied for both for low-power application, i.e. mobile device battery charging, and wireless electric vehicle charging, when the converter power is increased. To study the circuit as a linear and time-invariant system, usually the rectifier is modelled with an AC-resistance $R_{i}$, as shown in Fig. 2(c) [6], [7] and [8]. Compensation capacitors are used in WPT applications in both the primary and secondary windings of the WPT transformer to increase the efficiency and the capability of the system they are used in [17]. The compensation in the secondary winding enhances the power transfer capability of the WPT transformer while the primary compensation decreases the VA rating of the source side converter thereby ensuring power transfer at unity power factor. To increase the power transfer capability it is necessary that the system operates at the secondary resonance frequency, therefore, we have

$$
C_{2}=\frac{1}{\omega_{O}^{2} L_{2}}
$$

When operating at this frequency, the self-inductance of the secondary winding is fully compensated by the secondary compensation capacitance and, therefore, the impedance of the secondary as seen by the primary is purely resistive in nature. The input impedance seen from the sinusoidal voltage source is

$$
Z_{I N}=R_{1}+\frac{1}{j \omega C_{1}}+j \omega\left(L_{a}+M\right)+\frac{M^{2} \omega^{2}}{j \omega\left(L_{b}+M\right)+\frac{1}{j \omega C_{2}}+R_{2}+R_{L}}
$$

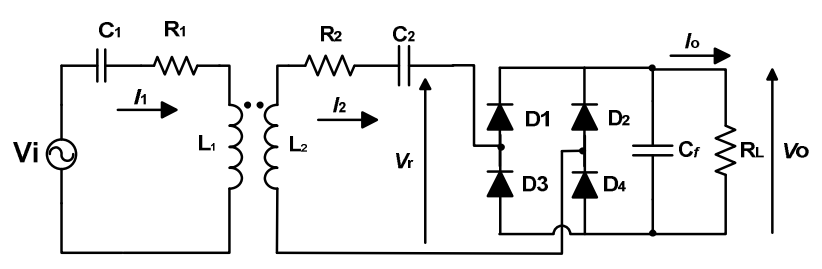

(a)

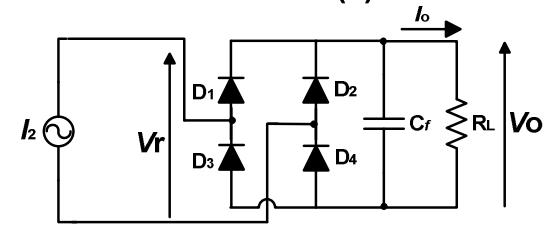

(b)

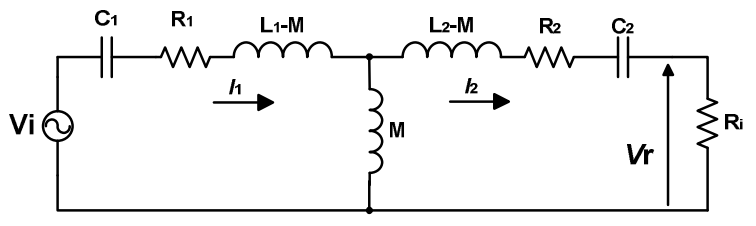

(c)

Fig. 2. Series-Series Compensation topology. (a) Class-D trasformer version of a current driven rectifier. (b) Series-Series Compensation with Class-D resonant rectifier. (c) Series-Series Compensation with rectifier equivalent resistance.

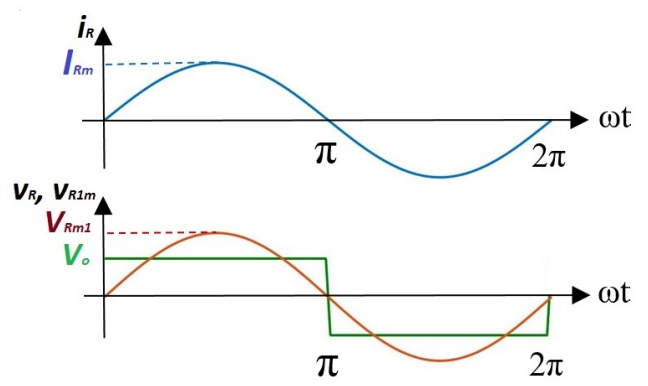

Fig. 3. Class D, current-driven, full-bridge rectifier input current and voltage.

The primary capacitance $C_{1}$ is designed to cancel the imaginary part of $Z_{I N}$. Therefore,

$$
C_{1}=\frac{1}{\omega_{O}^{2} L_{1}}
$$

The equation describing the circuit shown in Fig. 2(c) is

$$
\left[\begin{array}{c}
\bar{V}_{1} \\
0
\end{array}\right]=\left[\begin{array}{cc}
R_{1}+j\left(\omega L_{1}-\frac{1}{\omega C_{1}}\right) & -j \omega M \\
-j \omega M & R_{i}+R_{2}+j\left(\omega L_{2}-\frac{1}{\omega C_{2}}\right)
\end{array}\right)\left[\begin{array}{c}
\bar{I}_{1} \\
\bar{I}_{2}
\end{array}\right]
$$

The analytical expressions for the input current $I_{l}$ and the output current phasors are derived from (24) as follow 
$\bar{I}_{1}=\frac{R_{i}+R_{2}+j\left(\omega L_{2}-\frac{1}{\omega C_{2}}\right)}{\left[R_{1}+j\left(\omega L_{1}-\frac{1}{\omega C_{1}}\right)\right]\left[R_{i}+R_{2}+j\left(\omega L_{2}-\frac{1}{\omega C_{2}}\right)\right]+(\omega M)^{2}} \bar{V}_{1}$

$$
\bar{I}_{2}=\frac{j \omega M}{\left[R_{1}+j\left(\omega L_{1}-\frac{1}{\omega C_{1}}\right)\right]\left[R_{i}+R_{2}+j\left(\omega L_{2}-\frac{1}{\omega C_{2}}\right)\right]+(\omega M)^{2}} \bar{V}_{1}
$$

If the parasitic resistances of the primary and secondary coil are neglected, and, it is assumed that the circuit operates at the resonance, then (26) simplifies as

$$
\bar{I}_{2}=\frac{j \bar{V}_{1}}{\omega_{0} M}=I_{2} e^{j \varphi_{I_{2}}}
$$

It can be noted that the Series-Series compensation acts as a current-source, providing a current independent to the load. Therefore, the power delivered to the load is

$$
P_{R L}=R_{i} I_{O}^{2}=\frac{8}{\pi^{2}} \frac{V_{i}^{2}}{\omega^{2} M^{2}} R_{L}
$$

If the input voltage $V_{i}$ and the coupling coefficient $k$ are supposed constant (i.e. static electric vehicle wireless charging), the output power $P_{R L}$ is directly proportional with the load resistance $R_{L}$.

A generic bipole can be modelled as a resistive load $R_{i}$ in parallel with a non-linear bipole $Z_{D}$, as shown in Fig. 4. Under pure resistive load conditions $N$ is zero and therefore $Z_{D}$ can be neglected.

\section{RECTIFIER ANALYSIS INCLUDING CURRENT DISTORTION}

\section{A. Rectifier Circuit Analysis}

As shown in Fig. 3, the analysis presented in the previous section assumes that input current $i_{2}$ is sinusoidal. Unfortunately, current distortion occurs. For this reason, it can be useful consider the current distortion and its effect to the output power. It is also assumed that considering the rectifier input voltage first harmonic sinusoidal waveform results is a reasonable assumption to focus the analysis on the effects of current waveform distortion.

As shown in Fig. 5(a) and Fig.6(a), current distortion increase with load resistance $R_{i}$. The plots shown in Fig. 5 refer to a circuit with $R_{L}=10 \Omega$ and the Total Harmonic Distortion of current $i_{1}$ is $\mathrm{THD}_{\mathrm{I}}=5.99 \%$, while those shown in Fig. 6 refers to a circuit with $R_{L}=300 \Omega$, and $\mathrm{THD}_{\mathrm{I}}=42.65 \%$. Fourier series of the waveforms shown in Fig. 5(b) and Fig. 6(b) resulted in a nearly constant value of fundamental component RMS value $I_{I}$ for all the four considered waveforms, while higher order components RMS values increase in more distorted waveforms.

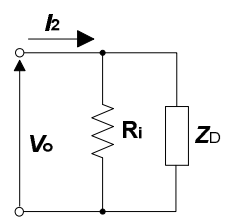

Fig. 4. Equivalent circuit including the "Non-Active" load.

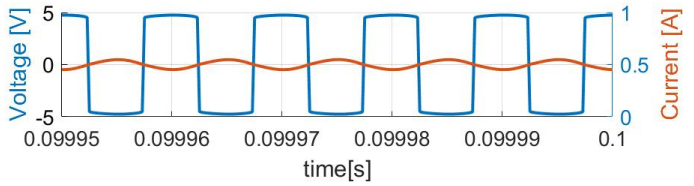

(a)

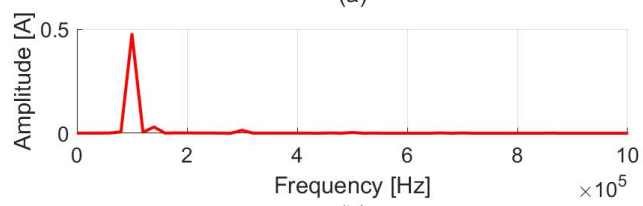

(b)

Fig. 5. Input rectifier waveform and input current Fourier Spectrum. (a) Input voltage rectifier $V_{r}$ (blue line), and input current rectifier $I_{2}$ (red line) with load resistance $R_{L}=10 \Omega$. (b) Input current $I_{2}$ Fast Fourier Transform.
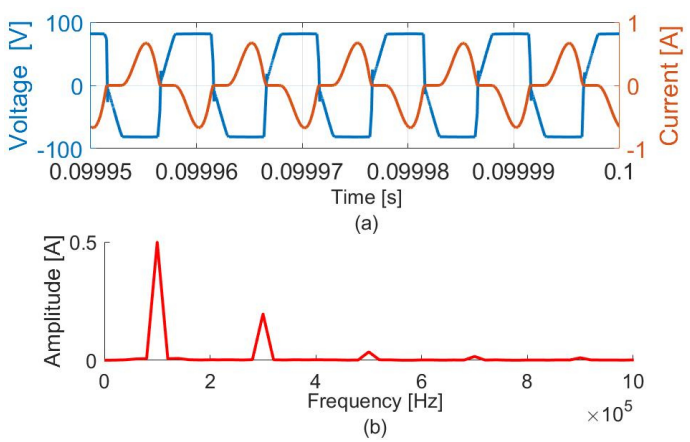

Fig. 6. Input rectifier waveform and input current Fourier Spectrum.

(a) Input voltage rectifier $\mathrm{Vr}$ (blue line), and input current rectifier $I_{2}$ (red line) with load resistance $R_{L}=300 \Omega$. (b) Input current $I_{2}$ Fast Fourier Transform.

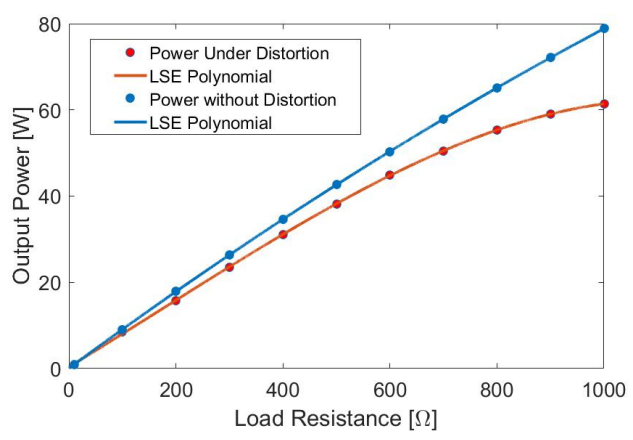

Fig. 7. Output Power without distortion (blue line) and Output Power from simulations (red line) varying load resistance.

By running several simulations, the red trace shown in Fig. 7 is derived. Note that blue plot refers to the output power one could derive by neglecting THD and using (28); for heavy loads (lower values of load resistance) the THD keeps low as shown 
in Fig. 8, and (28) allows for a reliable calculation of the power delivered to the load. For light loads the power delivered to the load is lower than calculated by using (28) and, see Fig. 8, the effects of current distortion are not negligible. Note that the simulations were performed utilizing component models including parasitics, therefore a certain level of disturbances that can affect the operation of a real circuit are included in the dotted plot shown in Fig. 8.

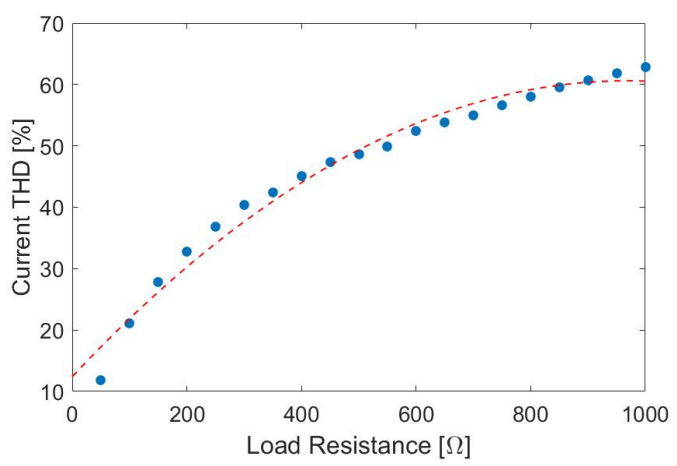

Fig. 8. Total harmonic distortion of the rectifier input current $I_{2}$ varying the load resistance $R_{L}$

\section{B. Case Study and Propsed Model at a Constant k}

The parameters used to design the circuit shown in Fig. 2 are provided in Table I.

TABLE I. SIMULATED CIRCUIT COMPONENT VALUES

\begin{tabular}{|c|c|c|}
\hline Parameter & Value & ESR \\
\hline \hline DC supply voltage $V_{I}$ & $15 \mathrm{~V}$ & - \\
Switching Frequency $f_{s}$ & $100 \mathrm{kHz}$ & - \\
Coupling Coefficient $k$ & 0.5 & - \\
Primary self-inductance $L_{1}$ & $100 \mu \mathrm{H}$ & $0.1 \Omega$ \\
Secondary self-inductance $L_{2}$ & $100 \mu \mathrm{H}$ & $0.1 \Omega$ \\
\hline
\end{tabular}

Using (21) and (23), the capacitance that resonate with the coupling inductor are calculated as $C_{1}=C_{2}=25.33 n \mathrm{~F}$. The output current is

$$
I_{2} \cong \frac{V_{1}}{\omega M}=0.3376 \mathrm{~A}
$$

Under the assumption of sinusoidal input current, the output power is

$$
P_{O} \cong R_{i} I_{2}^{2}=\frac{8}{\pi^{2}} R_{L} I_{2}^{2}=0.0924 R_{L}
$$

When the circuit is working at the resonance frequency $\omega=\omega_{0}$, the output power depends on the coupling coefficient $k$, the input voltage $V_{i}$ and the load resistance $R_{L}$. In wireless power transfer applications, the coupling coefficient can vary due to coil misalignment, while during charging and discharging cycle of a battery its equivalent impedance varies. In this section a procedure to find a model of the output power that consider the distortion and that is valid in a wide range of load resistance $R_{L}$, coupling coefficient $k$ and input voltage $V i$ variation is presented. The expression of the normalized output power $p$ is

$$
p=\frac{P_{\text {OUT }}}{P_{R L}}=\frac{P_{\text {OUT }}}{\frac{8}{\pi^{2}} R_{L} I_{2}^{2}}=\frac{P_{\text {OUT }}}{\frac{8}{\pi^{2}} R_{L}\left(\frac{V_{i}}{\sqrt{2} \omega M}\right)^{2}}=f\left(V_{i}, R_{L}, k\right)
$$

and it results the red trace shown in Fig.7. The output power can be modelled with a polynomial function of $R_{L}$

$$
P_{\text {OUT }}=f\left(R_{L}, R_{L}^{2}\right)=a_{2} R_{L}^{2}+a_{1} R_{L}
$$

where $a_{1}$ value is nearly the same of $8 V_{i}^{2} / \pi^{2} \omega^{2} M^{2}$ in (28).

By using Table I values, performing multiple simulations at different load resistance values and measuring the power derived to the load, a polynomial function for the real output power is derived as follows

$$
P_{\text {OUT }}=f\left(R_{L}, R_{L}^{2}\right)=-2.98 \times 10^{-5} R_{L}^{2}+0.093 R_{L}
$$

The output power is, than, given by

$$
P_{\text {OUT }}=I_{2}^{2}\left(R_{i}-\frac{k}{I_{2}^{2}} R_{L}^{2}\right)
$$

The distortion effects to the output power are considered by adding a non-linear bi-pole $Z_{D}$ in parallel with the rectifier equivalent impedance $R_{i}$, as shown in Fig. 4(a), where

$$
Z_{D}=\left[\frac{1}{k}\left(\frac{R_{i} I_{2}}{R_{L}}\right)^{2}\right]-R_{i}
$$

To increase the model accuracy of the an higher polynomial degree can be used. By using a $3^{\text {rd }}$ degree polynomial function, to the case study, the normalized output power $p$ is derived as

$$
p=-8.228 \cdot 10^{-10} R_{L}^{3}+1.177 \cdot 10^{-6} R_{L}^{2}-0.000559 \cdot R_{L}+0.974
$$

Finally, by removing the assumption that the rectifier input voltage is sinusoidal also other power components are determined for $k=0.5$ and $R_{L}=1 \mathrm{k} \Omega$. This requires the Fourier series derivation for both current $i_{2}(t)$ voltage $v_{r}(t)$ and the associated of the power expressions. As shown in Fig. 9 we have $P=66.01 \mathrm{~W}, Q=20.99$ VAR, $S=85.49 \mathrm{VA}, D=50.09 \mathrm{VA}$, $N=54.32 \mathrm{VA}$. The power $3 \mathrm{D}$ plot of the for the considered example is shown in Fig. 9.

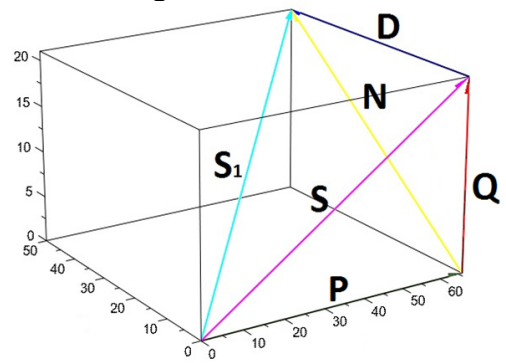

Fig. 9. Rectifier power components with $i_{2}(t)$ and $v_{r}(t)$ distorted at $V_{I}=15 \mathrm{~V}$, $k=0.5$ and $R_{L}=1 \mathrm{k} \Omega$.

\section{Result Extension at different $k$ values}

To obtain an expression for the output power valid for every winding distance, the analysis were performed also for other value of coupling coefficient $k$. As shown in Fig. 10, for a fixed 
value of load resistance, the distortion of the current $i_{2}$ increases with the coupling coefficient.

Coefficients $a_{1}$ and $a_{2}$ are expressed as functions of coupling factor $k$ as follow

$$
\begin{aligned}
& p=c_{1}(k) R_{L}+c_{2}(k) R_{L}^{2}+c_{3}(k) R_{L}^{3} \\
& =\left(0.016 k^{2}-0.023 k+0.0066\right) R_{L} \\
& +10^{-5}\left(-4.19 k^{2}+5.78 k-1.74\right) R_{L}^{2}+10^{-8}\left(2.39 k^{2}-3.25 k+0.96\right) R_{L}^{3}
\end{aligned}
$$

Table II lists the results of the proposed model. To validate the model, multiple simulations in a wide range of coupling coefficient $k$ and load resistance $R_{L}$ were performed, keeping input voltage constant $V_{i}=15 \mathrm{~V}$. Table II compares the simulations measured power $P_{M E A S U R E D}$ with the power obtained from the proposed approach $\left(P_{\text {OUT }}\right)$ and the power provided by (28) which is based on the assumption of pure sinusoid current.

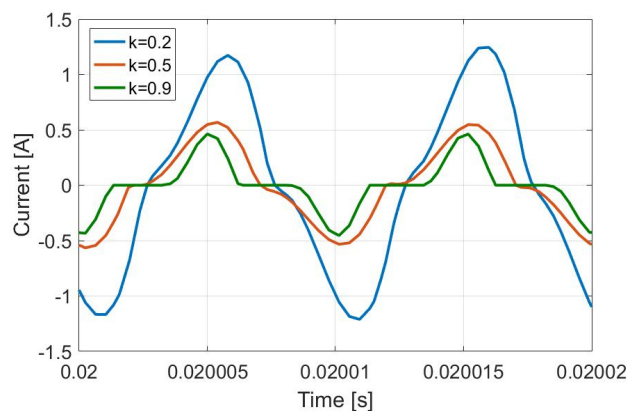

Fig. 10. Current distortion varying coupling coefficient for a fixed load resistance $R_{L}=100 \Omega$.

TABLE II

COMPARISON BETWEEN OUTPUT POWER MODELS

\begin{tabular}{|c|c|c|c|c|}
\hline $\begin{array}{c}\text { Coupling } \\
\text { Coefficient } \\
\boldsymbol{k}\end{array}$ & $\begin{array}{c}\text { Load } \\
\text { Resistance } \\
\boldsymbol{R}_{\boldsymbol{L}}(\mathbf{\Omega})\end{array}$ & $\begin{array}{c}\text { Measured Power } \\
\text { PMEASURED } \\
(\mathrm{W})\end{array}$ & $\begin{array}{c}\text { Proposed } \\
\text { Approach } \\
\boldsymbol{P}_{\text {OUT }}(\mathbf{W})\end{array}$ & $\begin{array}{c}\text { Conventional } \\
\text { Approach } \\
\boldsymbol{P}_{\text {RL }}(\mathbf{W})\end{array}$ \\
\hline \hline 0.3 & 10 & 2.52 & 2.31 & 2.56 \\
0.4 & 100 & 13.78 & 14.69 & 14.43 \\
0.5 & 200 & 15.79 & 18.51 & 18.48 \\
0.6 & 300 & 20.53 & 23.80 & 25.67 \\
0.7 & 600 & 21.23 & 25.20 & 28.30 \\
0.8 & 800 & 20.45 & 24.21 & 28.87 \\
0.9 & 1000 & 18.66 & 19.38 & 28.52 \\
\hline
\end{tabular}

\section{CONCLUSIONS}

A Series-Series compensation for wireless power transfer is presented. In many recent works, the rectifier is approximated with an equivalent AC-resistance. This approximation is based on the hypothesis of sinusoidal input current. Unfortunately, for light loads, the input current is distorted and the input current waveforms does not keep sinusoidal. As a result of the current distortion a lower time-average real power is delivered to the load. A new procedure to obtain and a new rectifier equivalent circuit are proposed in this paper to consider the current harmonics and improve the output power.

\section{REFERENCES}

[1] G. Tomy and D. Menon, "Power quality improvement strategy for nonlinear load in single phase systems," 2016 Intenational Conference on Electrical, Electronics and Optimizations techniques ICEEOT.

[2] K. Hata, T. Imura, and Y. Hori, "Analysis and experiment on harmonic current distortion in wireless power transfer system using a diode rectifier," Proc.of 41st Annual Conference of IEEE Industrial Electronics Society, November 9-12, 2015.

[3] E. H. Watanabe and M. Aredes, "Compensation of nonperiodic currents using the instantaneous power theory," in IEEE Power Engineering Soc. Summer Meeting, Seattle, WA, 2000, pp. 994-999.

[4] L. S. Czarnecki, "Non-periodic currents: their properties, identification and compensation fundamentals," in IEEE Power Engineering Soc. Summer Meeting, Seattle, WA, 2000, pp. 971-976.

[5] IEEE Std. 1459-2000, Definitions for the measurement of Electric quantities under sinusoidal, non-sinusoidal, balanced or unbalanced conditions, 2000.

[6] M. K. Kazimierczuk, RF Power Amplifiers, 2nd Ed., John Wiley Sons, Chichester, UK, 2014.

[7] M. K. Kazimierczuk and D. Czarkowski, Resonant Power Converters, 2nd Ed., John Wiley Sons, Hoboken, NJ, 2012.

[8] M. K. Kazimierczuk, "Class D current-driven rectifiers for resonant dc/dc converter applications," IEEE Transactions on Industrial Electronics, vol. IE-38, no. 10, pp. 344-354, October 1991.

[9] H. Sekiya, X. Wei, T. Nagashima, and M. K. Kazimierczuk, "Steadystate analysis and design of class-DE inverter at any duty ratio," IEEE Trans. Power Electron., vol. 30, no. 7, pp. 3685-3694, July 2015.

[10] A. Reatti, M. C. Piccirilli, M. K. Kazimierczuk, F. Grasso, A. Ayachit, L. Albertoni, J. Matteucci, "Analysis and design of full-bridge class-DE inverter at fixed duty cycle," Proc. of 42nd Annual Conference of IEEE Industrial Electronics Society, October 24-27,2016, Florence, Italy, pp. 56095614

[11] H. Sekiya, N. Sagawa, and M. K. Kazimierczuk, "Analysis of class DE amplifier with nonlinear shunt capacitances at any grading coefficient for high Q and $25 \%$ duty ratio," IEEE Trans. Power Electron., vol. 25, no. 4, pp. 924-932, Apr. 2010.

[12] C. Ekkavaradone, V. Chunkag, J. Kamon, and M. K. Kazimierczuk, "Class-D zero-current-switching rectifier as power factor corrector for lighting applications," IEEE Trans. Power ELectron., pp. 4938-4948, Oct. 2013.

[13] H. Sarnago, O. Lucia, A. Mediano, and J. M. Burdio, "Class-D/DE dual mode-operation resonant converter for improved-efficiency domestic induction heating system," IEEE Trans. Power Electron., vol. 28, no. 3, pp. 1274-1285, Mar. 2013.

[14] T. Suetsugu and M. K. Kazimierczuk, "Integration of class DE inverter for on-chip power supplies," Proc. IEEE IntI. Symp. Circ. Syst., Island of Kos, Greece, May 2006, pp. 3133-3136.

[15] A. Ayachit, A. Reatti, D. Saini, and M. K. Kazimierczuk, "Design of choke inductor in class-E ZVS power amplifier," Proc. of 42nd Annual Conference of IEEE Industrial Electronics Society, October 24-27,2016, Florence, Italy, pp. 5621-5626.

[16] A. Ayachit, F. Corti, F. Grasso, A. Reatti, D. Saini, and M. K. Kazimierczuk, "Design of class-E ZVS inverter with loosely-coupled transformer at fixed coupling coefficient", Proc. of 42nd Annual Conference of IEEE Industrial Electronics Society, October 24-27,2016, Florence, Italy, pp. $5627-5632$.

[17] X. Wei, H. Sekiya, S. Kuroiwa, T. Suetsugu, and M. K. Kazimierczuk, "Design of Class-E amplifier with MOSFET linear gate-to-drain and nonlinear drain-to-source capacitances," IEEE Trans. Circuits Syst.-I, vol. 58, no. 10, pp. 2556-2565, Oct. 2011.

[18] K. Inoue, T. Nagashima, X. Wei, and H. Sekiya, "Design of high-efficiency inductive-coupled wireless power transfer system with class-DE transmitter and class-E rectifier," Proc. of 39 the Annual Conference of IEEE Industrial Electronics Society, Novembre 10-13, 2013, Vienna, Austria, pp. 613 - 618 .

[19] M. K. Kazimierczuk, "Exact analysis of Class E tuned power amplifier with only one inductor and one capacitor in load network, "IEEE Journal of Solid-State Circuits, vol. SC-18, no. 2, pp. 214-221, April 1983. 
[20] M. K. Kazimierczuk and R. C. Cravens II, "Current-source parallelresonant dc/ac inverter with transformer," IEEE Transactions on Power Electronics, vol. PE-11, no. 2, pp. 275-284, March 1996.
[21] T. Suetsugu and M. K. Kazimierczuk, "Design procedure of Class E amplifier for off-nominal operation at 50\% duty ratio," IEEE Trans. Circuits and Systems, Part I, Regular Papers, vol. 53, no. 7, pp. 14681476, July 2 